\title{
Development of Plasticity Model Using Non-Associated Flow Rule For HCP Materials Including Zirconium for Nuclear Applications
}

Jeong-Whan Yoon Michael V. Glazoff

August 2013

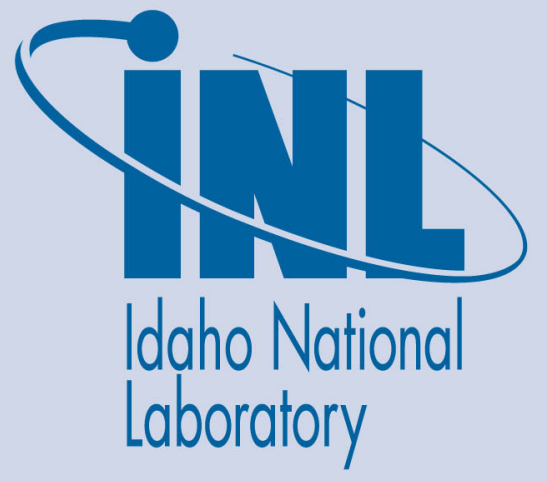

The INL is a U.S. Department of Energy National Laboratory operated by Battelle Energy Alliance 


\section{DISCLAIMER}

This information was prepared as an account of work sponsored by an agency of the U.S. Government. Neither the U.S. Government nor any agency thereof, nor any of their employees, makes any warranty, expressed or implied, or assumes any legal liability or responsibility for the accuracy, completeness, or usefulness, of any information, apparatus, product, or process disclosed, or represents that its use would not infringe privately owned rights. References herein to any specific commercial product, process, or service by trade name, trade mark, manufacturer, or otherwise, does not necessarily constitute or imply its endorsement, recommendation, or favoring by the U.S. Government or any agency thereof. The views and opinions of authors expressed herein do not necessarily state or reflect those of the U.S. Government or any agency thereof. 
Development of Plasticity Model Using Non-Associated Flow Rule for HCP Materials Including Zirconium for Nuclear Applications

\author{
Jeong-Whan Yoon and Jeong-Whan Yoon \\ Michael V. Glazoff
}

August 2013

\begin{abstract}
Idaho National Laboratory
UFD Campaign

Idaho Falls, Idaho 83415
\end{abstract}

http://www.inl.gov

Prepared for the

U.S. Department of Energy

Office of Nuclear Energy

Under DOE Idaho Operations Office

Contract DE-AC07-05ID14517 




\section{ABSTRACT}

In this report (prepared in collaboration with Prof. Jeong Whan Yoon, Deakin University, Melbourne, Australia) a research effort was made to develop a non-associated flow rule for zirconium. Since $\mathrm{Zr}$ is a hexagonally close packed (HCP) material, it is impossible to describe its plastic response under arbitrary loading conditions with any associated flow rule (e.g. von Mises). As a result of strong tension-compression asymmetry of the yield stress and anisotropy, zirconium displays plastic behavior that requires a more sophisticated approach.

Consequently, a new general asymmetric yield function has been developed which accommodates mathematically the four directional anisotropies along $0^{\circ}, 45^{\circ}, 90^{\circ}$, and biaxial, under tension and compression. Stress anisotropy has been completely decoupled from the r-value by using non-associated flow plasticity, where yield function and plastic potential have been treated separately to take care of stress and r-value directionalities, respectively. This theoretical development has been verified using $\mathrm{Zr}$ alloys at room temperature as an example as these materials have very strong SD (Strength Differential) effect. The proposed yield function reasonably well models the evolution of yield surfaces for a zirconium clock-rolled plate during in-plane and through-thickness compression. It has been found that this function can predict both tension and compression asymmetry mathematically without any numerical tolerance and shows the significant improvement compared to any reported functions.

Finally, in the end of the report, a program of further research is outlined aimed at constructing tensorial relationships for the temperature and fluence-dependent creep surfaces for Zr, Zircaloy-2, and Zircaloy-4. 


\section{CONTENTS}

ABSTRACT vii

1. INTRODUCTION

Error! Bookmark not defined.

2. DEVELOPMENT OF PLASTICITY MODEL USING NON-ASSOCIATED FLOW

RULE FOR HCP MATERIALS INCLUDING ZIRCONIUM FOR NUCLEAR

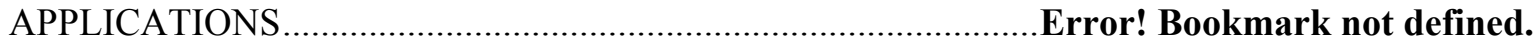

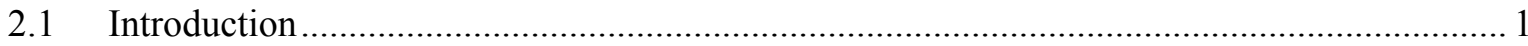

2.2 Plastic Potential for Non-Associated Flow Plasticity ......................................................... 1

2.3 A General Yield Function for the Tension Compression Asymmetry of Yield Stress

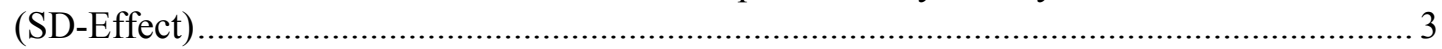

2.4 Extension of the Model for Orthotropic Metals ................................................................. 6

2.5 Modeling of Yield Surface Evolution for Zirconium Clock-Rolled Plate ............................. 7

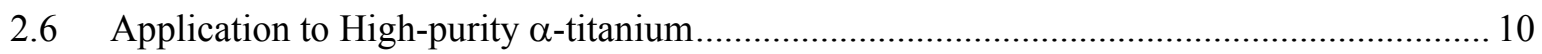

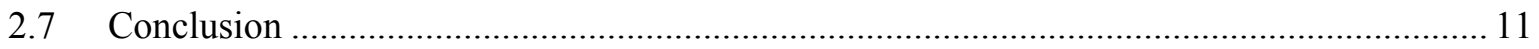

2.8 Recommendations and Path Forward: Temperature Dependent Creep Surface under

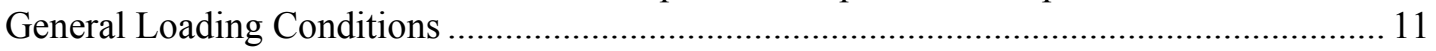

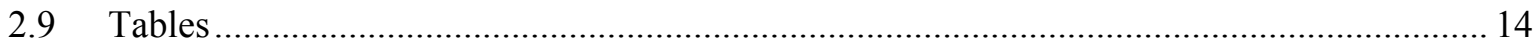

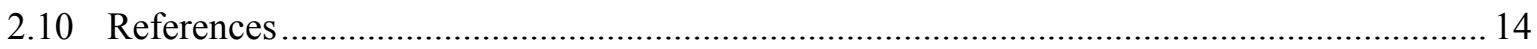

\section{FIGURES}

Figure 1. Effect of the pressure sensitive parameter $b$ on the yield surface of the proposed yield function constructed in the three-dimensional space of principal stresses: (a) negative $b$; (b) positive $b$

Figure 2. Effect of the pressure sensitive parameter $b$ on the yield surface of the proposed yield function constructed in the space of principal stresses under plane stress condition with $\sigma_{3}=0$.

Figure 3. Effect of the third stress invariant on the yield surface adjusted by the material parameter $c$ for materials under spatial loading.

Figure 4. Comparison of yield surface evolution predicted by the proposed yield function with those computed by the VPSC model for a zirconium clock-rolled plate during in-plane compression for various levels of pre-strain. (unit: MPa).

Figure 5. Comparison of yield surface evolution predicted by the proposed yield function with those computed by the VPSC model for a zirconium clock-rolled plate pre-strained in through-thickness compression for various levels of pre-strain. (unit: MPa).

Figure 6. Comparison the yield surfaces of high-purity $\alpha$-titanium constructed by the proposed yield function on the $\pi$-plane with experimental results (unit: MPa).

Figure 7. Comparison the yield surfaces of high-purity $\alpha$-titanium constructed by the proposed yield function under plane stress with experimental results (unit: MPa). 
Figure 8. Comparison of yield surface evolution predicted by the proposed yield function with those computed by the VPSC model for a zirconium clock-rolled plate during in-plane compression for various levels of pre-strain. (unit: MPa).

Figure 9. True stress behavior of Zirconium under compression under various high temperatures........... 13

Figure 10. Schematic view of a proposed general methodology.

\section{TABLES}

Table 1. Material constants in the proposed yield function in Eq. (23) for a zirconium clock-rolled plate during in-plane compression for various levels of pre-strain.

Table 2. Material constants in the proposed yield function in Eq. (23) for a zirconium clock-rolled plate during through-thickness compression for various levels of pre-strain. 


\section{Introduction}

Recently, hexagonal close-packed (HCP) metals attracted increasing efforts owning to the high ratio of strength to density. However, the plastic behavior of HCP metals shows noticeable difference between tension and compression, which is referred as the strength differential (SD) effect. SD effect is mainly due to the directional twinning of HCP metals. Cazacu and Barlat [1] restored the asymmetry of the third stress invariant to model SD effect of HCP metals. Cazacu et al. [2] developed another asymmetric yield function for HCP metals which covers the upper and lower bounds of SD effect for pressure insensitive metals. Plunkett et al. [3] (2006) applied the CPB'06 yield function to describe mechanical response of high-purity zirconium accounting anisotropic hardening. Nixon et al. [4] successfully modeled the anisotropic-asymmetric behavior of high-purity $\alpha$-titanium with the Cazacu-Barlat [1] yield function. Yoon et al. [5] employed the CPB'06 yield function for AZ31 sheet alloy with anisotropic hardening for simulation of axial crushing. Khan and $\mathrm{Yu}$ [6] experimentally investigate the anisotropic behavior of Ti-6Al-4V alloy both in tension and compression considering effect of strain rate and temperature. Khan et al. [7] also proposed a yield function with dependence on strain rate and temperature to model both the anisotropy and tension-compression asymmetric characteristics of Ti-6Al-4V alloy.

Advanced plasticity in modeling of plastic behavior of HCP metals poses three new challenges:

a) Constitutive models of plastic deformation should couple the effect of the through-thickness stress and be developed for metals under spatial loading;

b) Constitutive models can provide correct description of anisotropic behavior not only in tension but also in compression;

c) Constitutive functions are capable of modeling strong SD effect of HCP metals since employment of these metals is an efficient way for weight reduction.

However, the yield functions reviewed above do not fully provide satisfactory solutions for all of these challenges. In this work, a general yield function is proposed with dependence on the first, second and third invariants of the stress tensor. The yield function proposed is extended to an anisotropic form by introducing two different linear transformation tensors to the second and third invariants, respectively. Non-AFR is also used where the plastic strain directions are defined by a separate quadratic plastic potential and the directional hardenings are defined by the proposed yield function. So with this change it is possible to precisely reproduce the anisotropy in both yield and plastic strain behaviors, with the later assumed to be unaffected by plastic deformation. A constitutive model developed in this study substantially improves accuracy in the stress-strain prediction and it is expected to provide significantly more accurate results in applications where isotropic hardening is used and considered to be approximately valid as the case of HCP materials. Finally, the anisotropic form of the yield function proposed is utilized to model the yield surface evolution of a zirconium clock-rolled plate during in-plane and through-thickness compression to validate its flexibility.

\section{Development of Plasticity Model Using Non-Associated Flow Rule for HCP Materials Including Zirconium for Nuclear Applications}

\subsection{Plastic Potential for Non-Associated Flow Plasticity}

One of the most common models of metal deformation still widely used in industry is based on the quadratic plastic potential of the form proposed by Hill [8], which for plane stress conditions is often normalized for convenience to uniaxial tension along the 1-axis as follows, 


$$
\begin{gathered}
\bar{\sigma}_{p}(\vec{\sigma}) \equiv \sqrt{\sigma_{11}^{2}+\lambda_{p} \sigma_{22}^{2}-2 v_{p} \sigma_{11} \sigma_{22}+2 \rho_{p} \sigma_{12} \sigma_{21}} \\
\lambda_{p}=\frac{1+1 / r_{90}\left(\bar{\varepsilon}_{p}\right)}{1+1 / r_{0}\left(\bar{\varepsilon}_{p}\right)} \quad v_{p}=\frac{1}{1+1 / r_{0}\left(\bar{\varepsilon}_{p}\right)} \quad \rho_{p}=\frac{1 / r_{0}\left(\bar{\varepsilon}_{p}\right)+1 / r_{90}\left(\bar{\varepsilon}_{p}\right)}{1+1 / r_{0}\left(\bar{\varepsilon}_{p}\right)} \frac{1+2 r_{45}\left(\bar{\varepsilon}_{p}\right)}{2}
\end{gathered}
$$

The parameters, $r_{0}\left(\bar{\varepsilon}_{p}\right), r_{45}\left(\bar{\varepsilon}_{p}\right)$, and $r_{90}\left(\bar{\varepsilon}_{p}\right)$ are the ratios of the plastic strain rate across the width of a uniaxial tension test to the plastic strain rate through the thickness for tension at respectively, 0,45 , and 90 degrees to the rolling direction of the sheet coil aligned with the 1-axis of the coordinate system. The r-values are able to be defined as a function of effective plastic strain (non-constant values) as shown in Eq.1.

According to classical plasticity, the plastic potential is used in the flow rule to define the direction of the rate of change of all components of the plastic strain tensor,

$$
\frac{d E_{i j}^{(p)}}{d t}=\frac{\partial \bar{\sigma}_{p}(\vec{\sigma})}{\partial \sigma^{i j}} \dot{\lambda}
$$

where $\dot{\lambda}$ is the plastic compliance factor that controls the magnitude of the rate of change of the plastic strain tensor. Since the plastic potential is a linear homogeneous function of the stress, it follows from Eq. 2 , and the following sequence, that the rate of plastic work is equal to the product of the magnitude of the plastic potential and the plastic compliance factor,

$$
\frac{d w_{p}}{d t}=\sigma^{i j} \frac{d E_{i j}^{(p)}}{d t}=\sigma^{i j} \frac{\partial \bar{\sigma}_{p}(\vec{\sigma})}{\partial \sigma^{i j}} \dot{\lambda}=\bar{\sigma}_{p}(\vec{\sigma}) \dot{\lambda}
$$

Although it is not necessary to explicitly define the compliance factor for implementation in finite element analysis, it is useful to note that the quadratic form of the plastic potential, with the flow rule, also leads to a specific definition of the plastic compliance factor in terms of the parameters of the plastic potential and the components of the plastic strain rate tensor as follows,

$$
\dot{\lambda} \equiv \sqrt{\frac{\lambda_{p}\left(\dot{E}_{11}^{(p)}\right)^{2}+\left(\dot{E}_{22}^{(p)}\right)^{2}+2 v_{p} \dot{E}_{11}^{(p)} \dot{E}_{22}^{(p)}}{\lambda_{p}-v_{p}^{2}}+\frac{2}{\rho_{0}}\left(\dot{E}_{12}^{(p)}\right)^{2}}
$$

It is therefore a consequence of the flow rule and the linear homogeneous plastic potential, that the compliance factor is a specific linear homogeneous function of the rate of change of the plastic strain tensor, with parameters of this function determined solely by the parameters of the plastic potential. It is therefore reasonable to identify the plastic compliance as the rate of change of an effective plastic strain, and more to the point, the integral of which

$$
\bar{\lambda} \equiv \int \dot{\lambda} d t
$$

can be used as the work hardening variable in the stress-strain relation. This definition was adopted in the works on non-AFR in Stoughton and Yoon [9 - 11]. 


\subsection{A General Yield Function for the Tension Compression Asymmetry of Yield Stress (SD-Effect)}

For an isotropic material under spatial loading of a stress tensor denoted by $\boldsymbol{\sigma}$ or $\sigma_{i j}$, the stress state can be solely determined by three stress invariants expressed as follows:

$I_{1}=\operatorname{Tr}\left(\sigma_{i j}\right)=\sigma_{1}+\sigma_{2}+\sigma_{3}$

$J_{2}=\frac{1}{2} s_{i j} s_{i j}=\frac{1}{6}\left[\left(s_{1}-s_{2}\right)^{2}+\left(s_{2}-s_{3}\right)^{2}+\left(s_{3}-s_{1}\right)^{2}\right]$

$J_{3}=\operatorname{det}\left(s_{i j}\right)=s_{1} s_{2} s_{3}$

where $\sigma_{1}, \sigma_{2}$ and $\sigma_{3}$ are three principal stresses of the stress tensor of $\boldsymbol{\sigma}$ and $s_{1}, s_{2}$ and $s_{3}$ are three principal values of the stress deviator tensor of $\mathbf{s}$ or $s_{i j}$ computed by

$s_{i j}=\sigma_{i j}-\sigma_{m}=\sigma_{i j}-\frac{I_{1}}{3}$

with $\sigma_{m}$ as the mean stress.

Based on experimental results of uniaxial tension under a superimposed hydrostatic pressure for aluminum and steel alloys, Spitzig et al. [12] and Spitzig and Richmond [13] formulated the pressure sensitivity of metals by adding the first stress invariant to the von Mises stress as below:

$f\left(\sigma_{i j}\right)=\sqrt{3 J_{2}}+a I_{1}=\sigma_{\text {Mises }}+a I_{1}$

where $\sigma_{\text {Mises }}$ denotes the von Mises stress.

Drucker in 1952 [14] proposed a yield function to couple the effect of both the second and third invariants on yielding as below:

$f\left(\sigma_{i j}\right)=J_{2}^{3}-c J_{3}^{2}$

Cazacu and Barlat [1] restored the asymmetry of the third invariant in Eq. (11) to consider SD effect for pressure insensitive metals in a form of

$f\left(\sigma_{i j}\right)=J_{2}^{3 / 2}-c J_{3}$

Here a general yield function is formulated with three invariants as below:

$f\left(\sigma_{i j}\right)=a\left[b I_{1}+\left(J_{2}^{3 / 2}-c J_{3}\right)^{1 / 3}\right]$

This general yield function assumes a linear dependence of yielding on the first invariant according to experimental results of Spitzig et al. [12] and Spitzig and Richmond [13] and preserves the asymmetry of the third stress invariant to model SD effect of pressure insensitive metals. The material constants of $b$ and $c$ modulate the influence of the pressure and the third invariant on yielding of metals while the material parameter $a$ is determined by experiments used to characterize the strain hardening behavior of metals. Normally, the strain hardening behavior is measured from uniaxial tensile tests and accordingly the material constant $a$ is computed in a form of 
$a=\frac{1}{b+\left(\frac{1}{3 \sqrt{3}}-\frac{1}{27} c\right)^{1 / 3}}$

When the material constant $b$ is zero, Eq. (14) reduces to the Cazacu-Barlat'2004 yield function. The von Mises yield function is realized from Eq. (14) when both $b$ and $c$ disappear. Moreover, it is easy to prove that the material constant $b$ does not affect the convexity of the proposed yield function since the Hessian matrix of the proposed yield function is identical to that of the Cazacu-Barlat'2004 yield function. Therefore, the convexity of the proposed yield function in Eq. (14) is satisfied when $c \in[-3 \sqrt{3} / 4,3 \sqrt{3} / 4]$ which is the same as that for the Cazacu-Barlat'2004 yield function.

The effect of pressure sensitivity is modulated by the parameter $b$ as illustrated in Figs. 1 (a) and (b). It is observed that yield stresses increase with the mean stress when $b<0$, while raising the mean stress reduces the yield stresses when $b>0$. When $b=0$, yielding is independent of the first stress invariant. Effect of $b$ is also depicted for the yield surface under plane stress in Fig. 2. The ratio of tensile yield stress to compressive yield stress decreases as $b$ rises from a negative value to a positive one.

The material parameter $c$ adjusts effect of the third stress invariant on yielding. The material constant $b$ is set to zero to graphically illustrate influence of $c$ on the yield surface since the three-dimensional yield surface can be plotted on the $\pi$-plane in Fig. 3 for pressure insensitive materials. It is observed that the yield surface is convex when $c \in[-3 \sqrt{3} / 4,3 \sqrt{3} / 4]$. In addition, SD effect is correctly modeled without the effect of the pressure sensitivity.

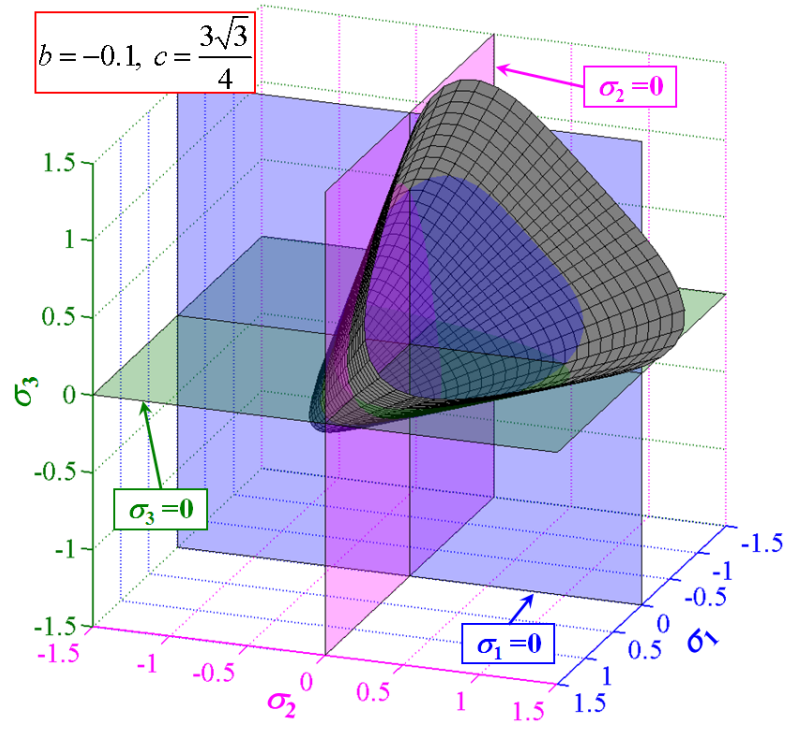

(a)

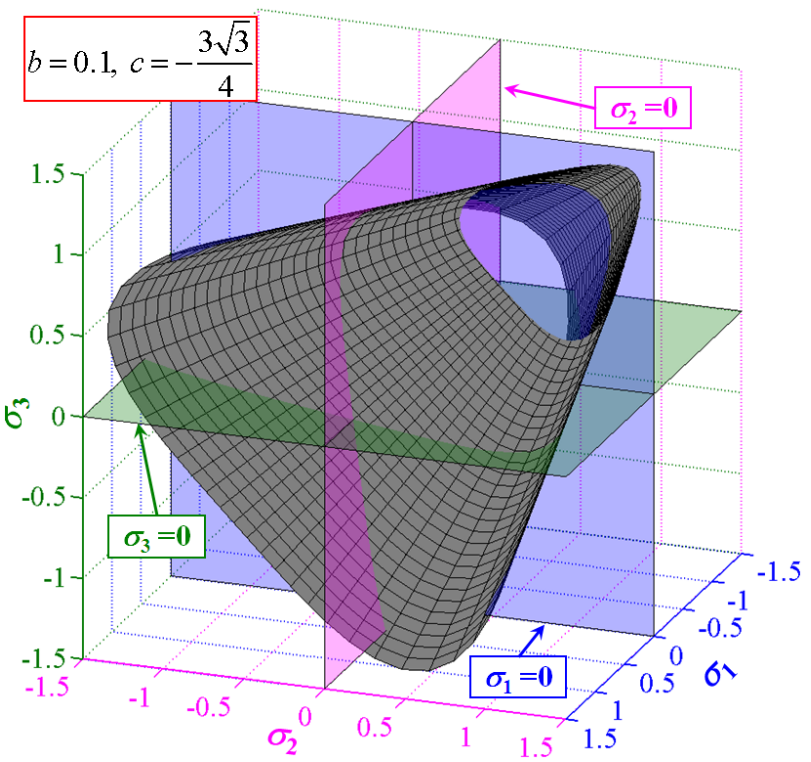

(b)

Figure 1. Effect of the pressure sensitive parameter $b$ on the yield surface of the proposed yield function constructed in the three-dimensional space of principal stresses: (a) negative $b$; (b) positive $b$. 


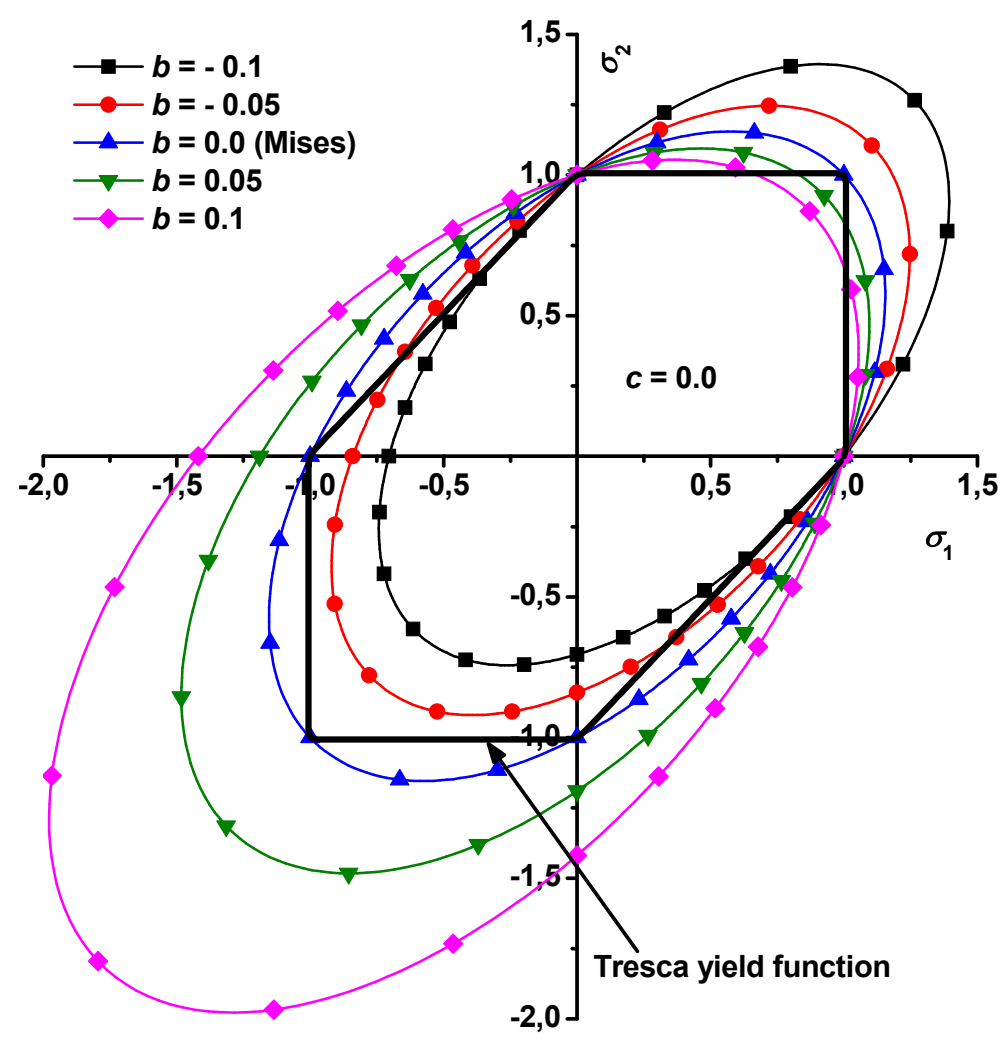

Figure 2 Effect of the pressure sensitive parameter $b$ on the yield surface of the proposed yield function constructed in the space of principal stresses under plane stress condition with $\sigma_{3}=0$

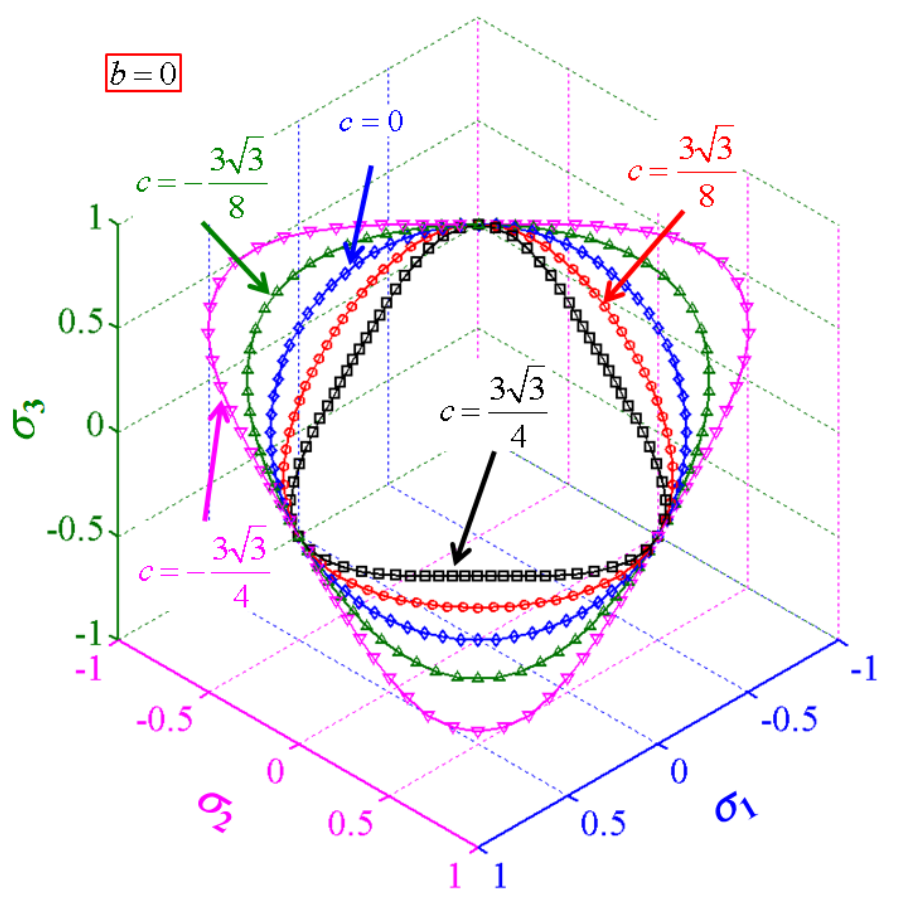

Figure 3 Effect of the third stress invariant on the yield surface adjusted by the material parameter $c$ for materials under spatial loading 


\subsection{Extension of the Model for Orthotropic Metals}

The yield function proposed can be extended to describe the anisotropic plastic deformation for orthotropic metals in a form of

$f\left(\sigma_{i j}\right)=\tilde{I}_{1}+\left(J_{2}^{\prime 3 / 2}-J_{3}^{\prime \prime}\right)^{1 / 3}$

with

$\tilde{I}_{1}=h_{x} \sigma_{x x}+h_{y} \sigma_{y y}+h_{z} \sigma_{z z}$

$J_{2}^{\prime}=\frac{1}{2} \mathbf{s}^{\prime}: \mathbf{s}^{\prime}=\frac{1}{6}\left[\left(s_{1}^{\prime}-s_{2}^{\prime}\right)^{2}+\left(s_{2}^{\prime}-s_{3}^{\prime}\right)^{2}+\left(s_{3}^{\prime}-s_{1}^{\prime}\right)^{2}\right]$

$J_{3}^{\prime \prime}=\operatorname{det}\left(\mathbf{s}^{\prime \prime}\right)=s_{1}^{\prime \prime} s_{2}^{\prime \prime} s_{3}^{\prime \prime}$

where $\tilde{I}_{1}$ represents the anisotropic-weighted first stress invariant for pressure sensitivity, $J_{2}^{\prime}$ is the second stress invariant of an isotropic plastic equivalent (IPE) transformed stress tensor of $\mathbf{s}^{\prime}$, and $J_{3}^{\prime \prime}$ is the third stress invariant of another IPE transformed stress tensor of $\mathbf{s}^{\prime \prime} . s_{1}^{\prime}, s_{2}^{\prime}$ and $s_{3}^{\prime}$ are three principal values of $\mathbf{s}^{\prime}$ while $s_{1}^{\prime \prime}, s_{2}^{\prime \prime}$ and $s_{3}^{\prime \prime}$ are three principal values of $\mathbf{s}^{\prime \prime}$. The axes of $\mathrm{x}, \mathrm{y}$ and $\mathrm{z}$ represent rolling direction (RD), transverse direction (TD), and normal direction (ND) of cold rolled metals, respectively. Two IPE stress tensors of $\mathbf{s}^{\prime}$ and $\mathbf{s}^{\prime \prime}$ are transformed from the stress tensor $\boldsymbol{\sigma}$ by two fourth-order linear transformation tensors of $\mathbf{L}^{\prime}$ and $\mathbf{L}^{\prime \prime}$ as follows:

$\mathbf{s}^{\prime}=\mathbf{L}^{\prime} \boldsymbol{\sigma}, \mathbf{s}^{\prime \prime}=\mathbf{L}^{\prime \prime} \boldsymbol{\sigma}$

with

$\mathbf{L}^{\prime}=\left[\begin{array}{cccccc}\left(c_{2}^{\prime}+c_{3}^{\prime}\right) / 3 & -c_{3}^{\prime} / 3 & -c_{2}^{\prime} / 3 & 0 & 0 & 0 \\ -c_{3}^{\prime} / 3 & \left(c_{3}^{\prime}+c_{1}^{\prime}\right) / 3 & -c_{1}^{\prime} / 3 & 0 & 0 & 0 \\ -c_{2}^{\prime} / 3 & -c_{1}^{\prime} / 3 & \left(c_{1}^{\prime}+c_{2}^{\prime}\right) / 3 & 0 & 0 & 0 \\ 0 & 0 & 0 & c_{4}^{\prime} & 0 & 0 \\ 0 & 0 & 0 & 0 & c_{5}^{\prime} & 0 \\ 0 & 0 & 0 & 0 & 0 & c_{6}^{\prime}\end{array}\right]$

$\mathbf{L}^{\prime \prime}=\left[\begin{array}{cccccc}\left(c_{2}^{\prime \prime}+c_{3}^{\prime \prime}\right) / 3 & -c_{3}^{\prime \prime} / 3 & -c_{2}^{\prime \prime} / 3 & 0 & 0 & 0 \\ -c_{3}^{\prime \prime} / 3 & \left(c_{3}^{\prime \prime}+c_{1}^{\prime \prime}\right) / 3 & -c_{1}^{\prime} / 3 & 0 & 0 & 0 \\ -c_{2}^{\prime \prime} / 3 & -c_{1}^{\prime \prime} / 3 & \left(c_{1}^{\prime \prime}+c_{2}^{\prime \prime}\right) / 3 & 0 & 0 & 0 \\ 0 & 0 & 0 & c_{4}^{\prime \prime} & 0 & 0 \\ 0 & 0 & 0 & 0 & c_{5}^{\prime \prime} & 0 \\ 0 & 0 & 0 & 0 & 0 & c_{6}^{\prime \prime}\end{array}\right]$

$\boldsymbol{\sigma}^{T}=\left(\begin{array}{llllll}\sigma_{x x} & \sigma_{y y} & \sigma_{z z} & \sigma_{y z} & \sigma_{x z} & \sigma_{x y}\end{array}\right)$

In the extended anisotropic form of the yield function proposed, the material constants of $a, b$ and $c$ in the isotropic form of the yield function are implicitly coupled by $h_{x}, h_{y}, h_{z}$ and anisotropic parameters in two fourth-order linear transformation tensors of $\mathbf{L}^{\prime}$ and $\mathbf{L}^{\prime \prime}$. 
The anisotropic pressure sensitivity parameters of $h_{x}, h_{y}$ and $h_{z}$ are computed by uniaxial tensile tests in rolling direction (RD), transverse direction (TD), and normal direction (ND) under different hydrostatic pressure. Here the pressure sensitivity is assumed to be isotropic for the simplicity purpose. That is, $h_{x}=h_{y}=h_{z}=b$. Then the anisotropic yield function reduces to a form of

$f\left(\sigma_{i j}\right)=b I_{1}+\left(J_{2}^{\prime 3 / 2}-J_{3}^{\prime \prime}\right)^{1 / 3}$

\subsection{Modeling of Yield Surface Evolution for Zirconium Clock-Rolled Plate}

The yield function proposed in Eq. (23) is employed to illustrate the yield surface evolution of a zirconium clock-rolled plate during in-plane and through-thickness compression for various levels of pre-strains. The evolution of yield surfaces is reported by Plunkett et al. [15] using the visco-plastic self-consistent (VPSC) polycrystalline model of Lebensohn and Tomé [16]. In the VPSC model, the initial texture was obtained from experiments. The deformation mechanisms are assumed to be composed of prismatic $<\mathrm{a}>$-slip, pyramidal $<\mathrm{c}+\mathrm{a}>$-slip and tensile twining. The slip and twinning parameters (i.e. critical stresses, hardening coefficients and rate sensitivity exponent) are referred after Tomé et al. [17].

Effect of texture evolution is investigated on the yielding behavior of the zirconium clock-rolled plate using the VPSC polycrystalline model. Yield stresses are depicted as the symbols in Fig. 4 during in-plane compression for various levels of $1 \%, 5 \%, 25 \%, 35 \%, 45 \%$ and $60 \%$. For each level of pre-strain, the material constants of the proposed yield function in Eq. (23) are optimized to match with yield stresses with minimum errors as summarized in Table 1. With the material constants optimized in Table 1, the yield surfaces are constructed for different levels of pre-strains and compared with the stresses computed by the VPSC polycrystalline model in Fig. 4. The comparison reveals that the proposed yield function accurately describes the yielding behavior of the zirconium clock-rolled plate for various levels of pre-strain in in-plane compression. 


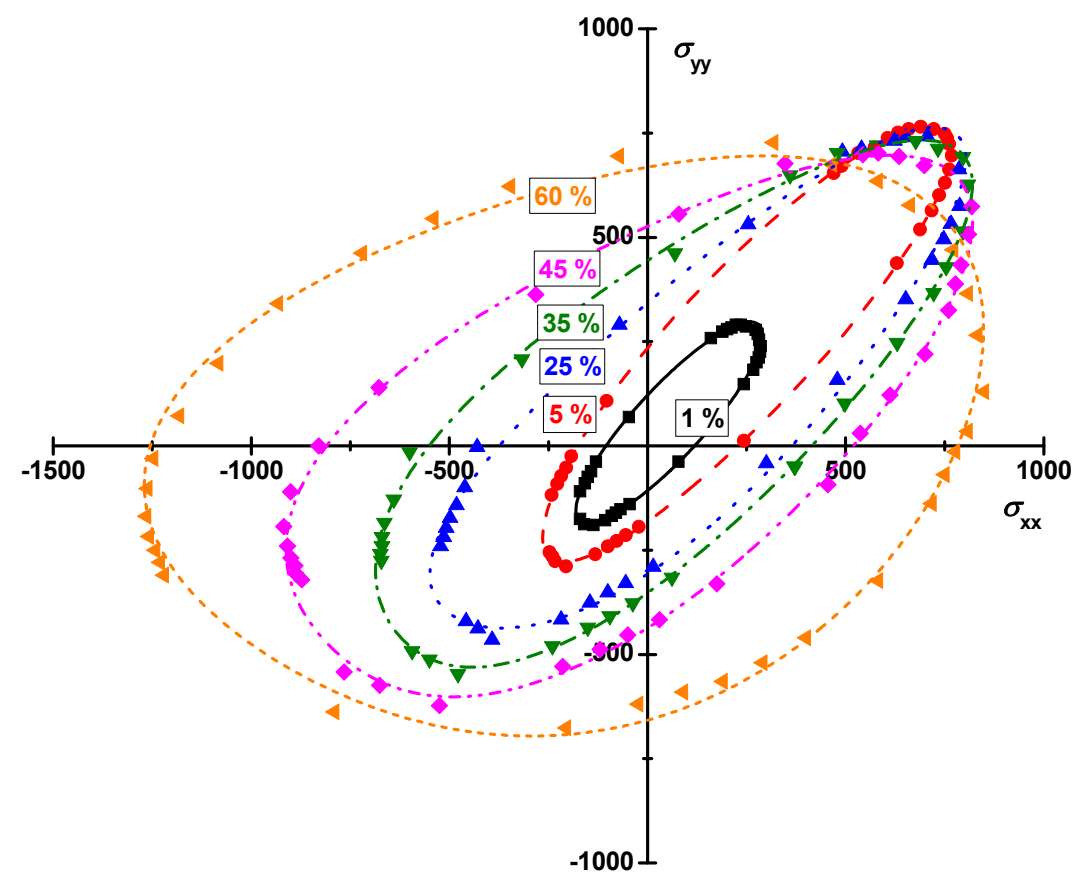

Figure 4 Comparison of yield surface evolution predicted by the proposed yield function with those computed by the VPSC model for a zirconium clock-rolled plate during in-plane compression for various levels of pre-strain. (units: MPa)

In addition, yield stresses of the identical metals are computed by the VPSC polycrystalline model during through-thickness compression for different levels of pre-strains $(0.2 \%, 1 \%, 5 \%, 25 \%$ and 35 $\%)$ as illustrated by symbols in Fig. 5. 


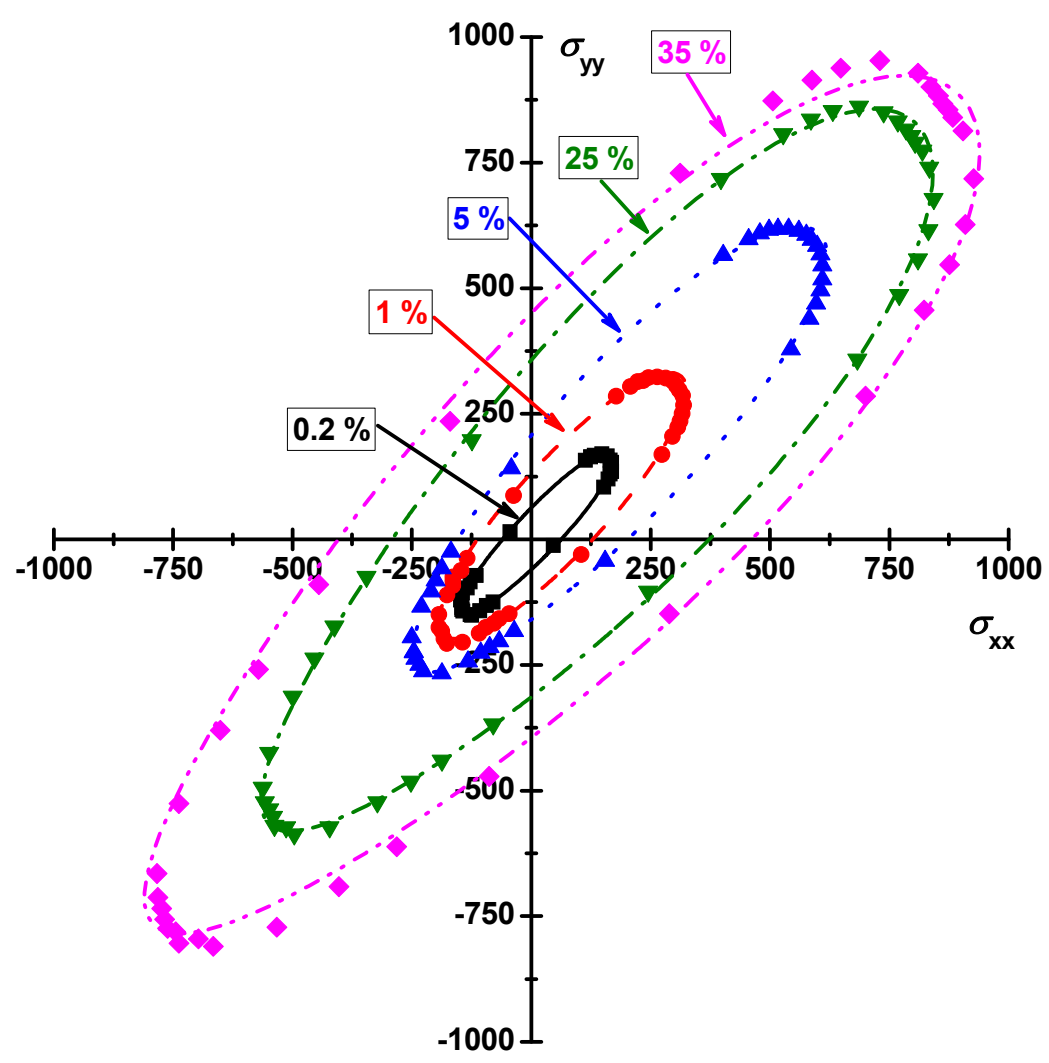

Figure 5 Comparison of yield surface evolution predicted by the proposed yield function with those computed by the VPSC model for a zirconium clock-rolled plate pre-strained in through-thickness compression for various levels of pre-strain. (units: $\mathrm{MPa}$ )

These yield stresses are utilized to optimize the material constants of the proposed yield function as listed in Table 2. The yield surfaces are predicted by the yield function in Eq. (23) with the material constants optimized. The yield surfaces predicted are compared with those computed by the VPSC model. It is obvious that the proposed yield function accurately models the evolution of the yield surfaces during through-thickness compression for different pre-strain levels. 


\subsection{Application to High-purity $\alpha$-titanium}

Titanium alloys are widely used in aerospace engineering, energy and automotive industries owning to their attractive mechanical properties of elevated strength-to-density ratio, exceptional corrosion and erosion resistance and excellent structural efficiency even at high temperatures. Titanium alloys are also a promising material in body implants and prosthetic devices because of the inherent non-reactivity with tissue and bone: nontoxic, non-allergenic and fully biocompatible. Thus, the proposed yield function is applied to describe plastic behavior of a high purity $\alpha$-titanium, Nixon et al. [4]. Yielding of this metal is assumed to be insensitive to hydrostatic pressure because there is no experimental result from uniaxial tensile tests under different hydrostatic pressure indicating that hydrostatic pressure affects plastic deformation of titanium alloys. Moreover, SD effect of high-purity $\alpha$-titanium is not severe and can be successfully modeled by the proposed yield function without pressure sensitivity. Therefore, the material constant of $b$ is set to zero for high-purity $\alpha$-titanium. Taking the advantage of pressure insensitivity of high-purity $\alpha$-titanium, the three-dimensional yield surfaces are constructed on the $\pi$-plane by the yield function proposed for various amount of plastic strain: $0 \%, 5 \%, 10 \%$ and $20 \%$. The constructed yield surfaces are compared with experimental data points as depicted in Fig. 3-6. Besides, comparison of the yield surfaces is also carried out under plane stress conditions in Fig. 3-7. The comparison in both spatial loading and plane stress demonstrates that the yield function proposed accurately models the orthotropic behavior of the high-purity $\alpha$-titanium not only in tension but also in compression.

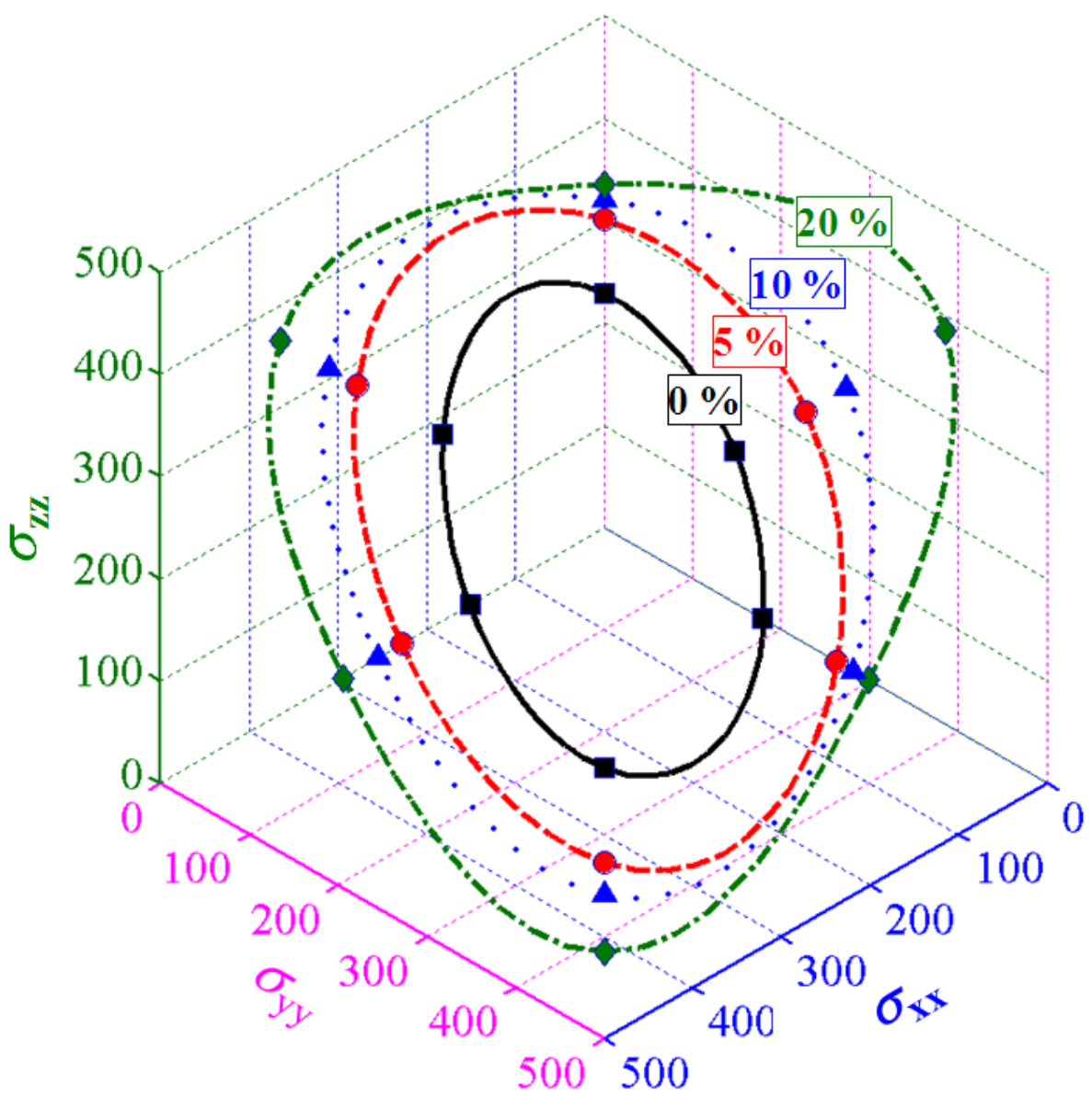

Figure 6 Comparison the yield surfaces of high-purity $\alpha$-titanium constructed by the proposed yield function on the $\pi$-plane with experimental results (units: MPa) 


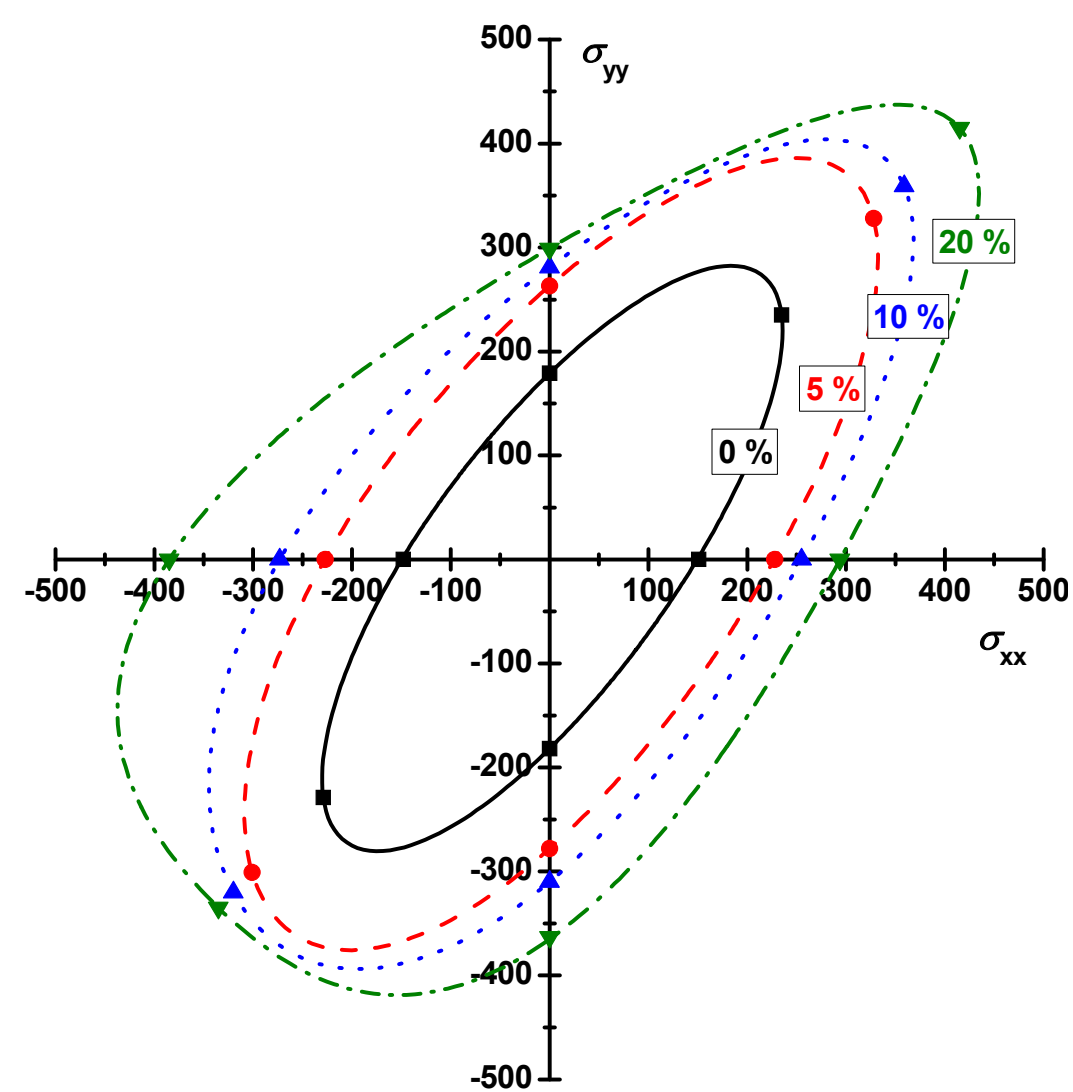

Figure 7 Comparison the yield surfaces of high-purity $\alpha$-titanium constructed by the proposed yield function under plane stress with experimental results (unit: MPa).

\subsection{Conclusions}

A new general yield function based on non-associated flow plasticity is proposed to extend symmetric yield functions to consider the SD effect for incompressible sheet metals. The SD effect is coupled by considering the hardening curves in both tension and compression. The new yield function is proven to be able to describe both anisotropy and asymmetry of sheet metals from the comparison of predicted yield surfaces for HCP metals with strong SD effect. The anisotropic form of the proposed yield function is validated to be capable of modeling evolution of yield surfaces for a zirconium clock-rolled plate during in-plane and through-thickness compression for various levels of pre-strain.

\subsection{Recommendations and Path Forward: Temperature Dependent Creep Surface under General Loading Conditions}

Constitutive laws in materials generally consist of a state equation and evolution equations. The state equation describes the relationship between the strain rate $\dot{\varepsilon}$, stress $\sigma$, temperature $T$ and state variables $\mathrm{x}^{\mathrm{k}}$, which represents the microstructural state of the material. The porosity of a material and a measure of the accumulated plastic deformation such as the effective strain or the dislocation density are a few examples of the variables $\mathrm{x}^{\mathrm{k}}$. The state equation can be expressed mathematically, for instance in a scalar form for uniaxial deformation, as 
$\dot{\varepsilon}=\dot{\varepsilon}\left(\sigma, \mathrm{T}, \mathrm{x}^{\mathrm{k}}\right)$

The evolution equations describe the development of the microstructure through the change of the state variables and can take the form

$\dot{\mathrm{x}}^{\mathrm{k}}=\dot{\mathrm{x}}^{\mathrm{k}}\left(\sigma, \mathrm{T}, \mathrm{x}^{\mathrm{k}}\right)$

In Phase-I, the evolution equation for $\mathrm{Zr}$. has been derived at the room temperature as shown in Figures 3-8. Then, the evolution model was only the function of stress (not a function of temperature)

$$
\dot{x}^{k}=\dot{x}^{k}\left(\sigma, x^{k}\right)
$$

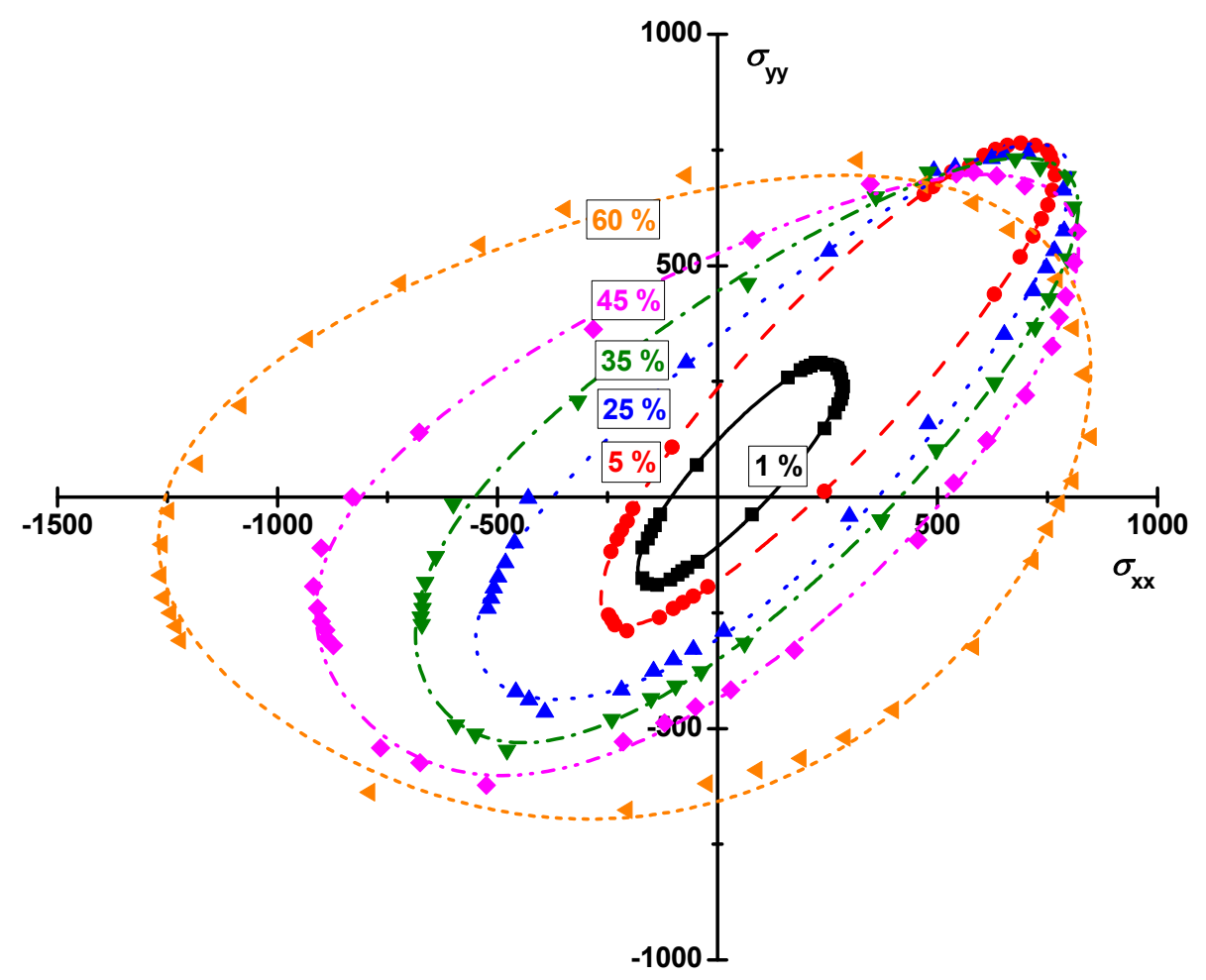

Figure 8 Comparison of yield surface evolution predicted by the proposed yield function with those computed by the VPSC model for a zirconium clock-rolled plate during in-plane compression for various levels of pre-strain. (units: MPa)

Eq.(3-26) was derived based on non-associated flow plasticity. Also, the VPSC (Visco-Plastic Self Consistent) model utilizing texture measurement was also utilized to replace the extensive experimental testing to calibrate the proposed model.

In Phase-II, a temperature dependent creep evolution model for Zr. alloy is proposed to be developed. As shown in Fig.2, Zr. shows a dramatic decrease of yield stress by increasing temperature. If the critical stress to initiate cracking of $\mathrm{Zr}$. is defined, cracking is assumed to be occurred with

$$
\Delta T \geq \frac{2 \sigma_{c r}(1-v)}{\alpha E}
$$




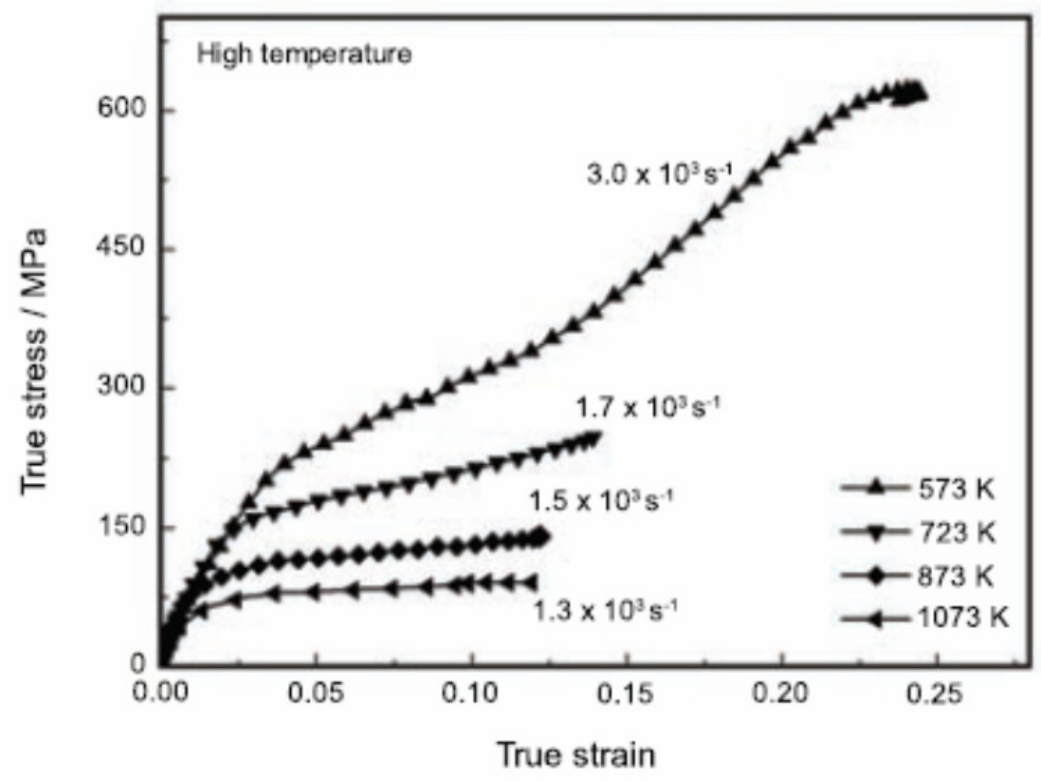

Figure 9 True stress behavior of Zirconium under compression under various high temperatures

Eq.(27) is commonly accepted in nuclear industry. However, since Zr. shows very strong asymmetric and anisotropic plastic behaviors, the simple scalar equation to connect Zr. creep fracture only with temperature and crystal stress is not reliable. The creep criteria in Eq. (27) must be represented in a continuous tensor space with temperature dependency as the initiation of creep behavior.

For this purpose, in-situ texture data with various temperatures should be measured for the VPSC crystal plasticity model to calibrate the time dependent coefficients of the constitutive model. Also, one directional in-situ tensile or compression testing under each temperature is recommended for a reference point. Then, it is able to generate a temperature dependent creep model like Eq.2 and to predict the precise creep behavior of $\mathrm{Zr}$, alloy. The merit of this approach is to incorporate minimum experimental data with the help of crystal plasticity modeling. Also, it is allowed to generate the complete creep space under a general loading condition at any temperature.

\section{Input data:}

- Tensile testing along one direction for selected several temperatures

- Texture date for selected several temperature.

\section{Predictions:}

(VPSC Polycrystal Model for the selected temperatures)

- Directional properties for any loading condition

- Evolution of r-values with respect to effective plastic strain

- Discrete strain-rate potential.

Non-Associated Continuum Model)

- Yield Surface Evolution as the function of temperature and stress triaxiality

- Dual Creep Surface (Strain-rate potential) from yield surface evolution. 
The schematic diagram to explain input and output is presented in Fig.10.

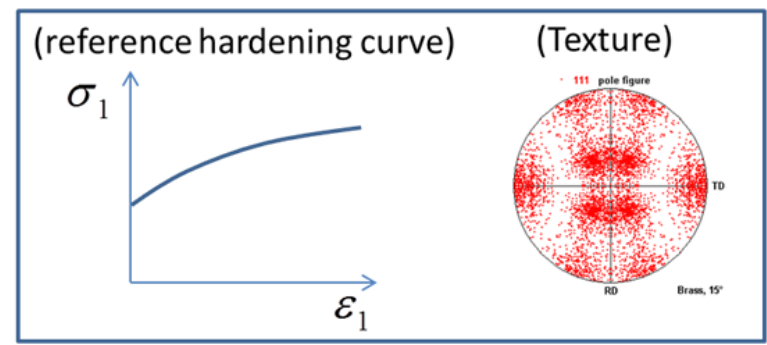

(Input for a given $\mathrm{T}^{*}$ )

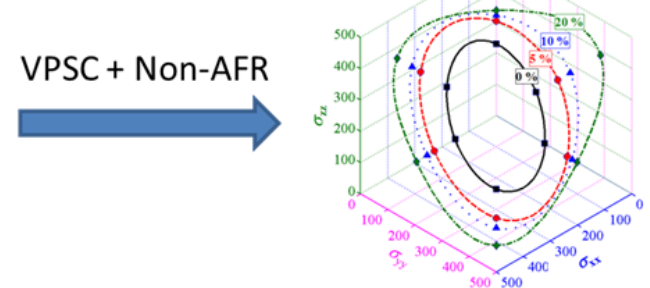

$$
\bar{\sigma}=(\underline{\underline{\sigma}})
$$

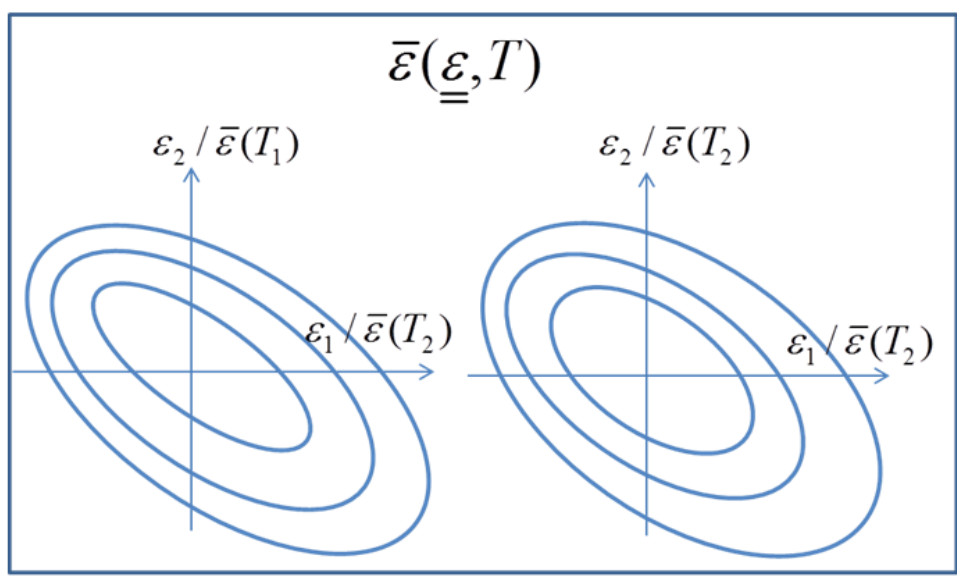

(Output)

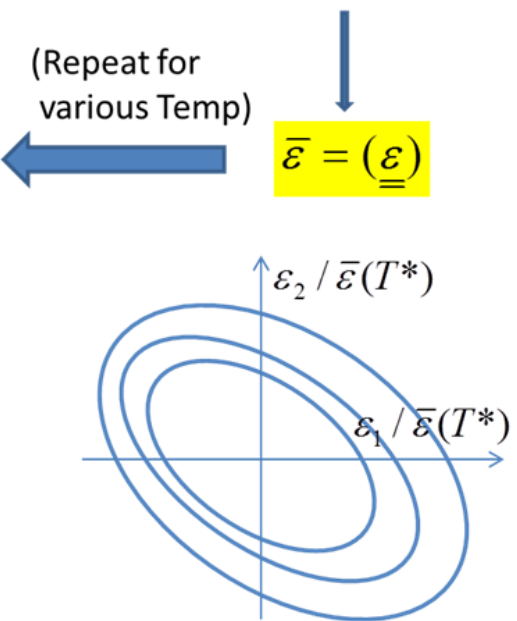

Figure 10 Schematic view of a proposed general methodology

\subsection{Tables}

Table 1 Material constants in the proposed yield function in Eq. (23) for a zirconium clock-rolled plate during in-plane compression for various levels of pre-strain

\begin{tabular}{cccccccc}
\hline Pre-strain & $b$ & $c_{1}^{\prime}$ & $c_{2}^{\prime}$ & $c_{3}^{\prime}$ & $c_{1}^{\prime \prime}$ & $c_{2}^{\prime \prime}$ & $c_{3}^{\prime \prime}$ \\
\hline $1 \%$ & -0.0608 & 0.8296 & 0.9253 & 2.3893 & -0.0242 & -0.0802 & 0.0553 \\
$5 \%$ & -0.1410 & 0.9767 & 1.1056 & 2.7774 & -1.0342 & 1.3298 & 0.3355 \\
$25 \%$ & -0.0100 & 1.3992 & 0.8097 & 2.2170 & -3.7006 & -0.3056 & 0.2823 \\
$35 \%$ & -0.0563 & 1.4783 & 0.8918 & 2.2120 & 1.1077 & -0.9910 & -2.9780 \\
$45 \%$ & -0.0319 & 1.8039 & 0.9850 & 1.9848 & 0.6250 & -0.7562 & -3.6015 \\
$60 \%$ & 0.0003 & 2.6681 & 1.5868 & 1.3944 & 0.8682 & -1.8095 & -1.6691 \\
\hline
\end{tabular}

Table 2 Material constants in the proposed yield function in Eq. (23) for a zirconium clock-rolled plate during through-thickness compression for various levels of pre-strain 


\begin{tabular}{cccccccc}
\hline Pre-strain & $b$ & $c_{1}^{\prime}$ & $c_{2}^{\prime}$ & $c_{3}^{\prime}$ & $c_{1}^{\prime \prime}$ & $c_{2}^{\prime \prime}$ & $c_{3}^{\prime \prime}$ \\
\hline $0.2 \%$ & -0.0178 & 0.5946 & 0.6074 & 2.3372 & 0.4017 & -0.7646 & 0.2295 \\
$1 \%$ & -0.0746 & 0.9357 & 1.0463 & 2.7183 & -0.0008 & -0.0450 & 0.0245 \\
$5 \%$ & -0.1379 & 0.9066 & 1.0312 & 2.6937 & 0.5818 & 1.1102 & 0.8839 \\
$25 \%$ & -0.0685 & 0.8530 & 0.9600 & 2.5815 & -1.6096 & 1.1486 & 1.6666 \\
$35 \%$ & -0.0621 & 0.9858 & 0.8798 & 2.4899 & 0.9359 & 1.0635 & -1.3575 \\
\hline
\end{tabular}

\subsection{References}

1. Cazacu, O., Barlat, F., A criterion for description of anisotropy and yield differential effects in pressure-insensitive metals, International Journal of Plasticity 20, 2027-2045 (2004)

2. Cazacu, O., Plunkett, B., Barlat, F., Orthotropic yield criterion for hexagonal closed packed metals, International Journal of Plasticity 22, 1171-1194 (2006)

3. Plunkett, B., Lebensohn, R.A., Cazacu, O., Barlat, F., Anisotropic yield function of Hexagonal materials taking into account texture development and anisotropic hardening, Acta Materialia 54, 4159-4169 (2006)

4. Nixon, M.E., Cazacu, O., Lebensohn, R.A., Anisotropic response of high-purity $\alpha$-titanium: experimental characterization and constitutive modeling International Journal of Plasticity 26, 516-532 (2010)

5. Yoon, J.H., Cazacu, O., Mishra, R.K., Constitutive modeling of AZ31 sheet alloy with application to axial crushing, Materials Science \& Engineering A 565, 203-212 (2013)

6. Khan, S.K., Yu, S., Deformation induced anisotropic responses of Ti-6Al-4V alloy. Part I: experiments, International Journal of Plasticity, 38, 1-13 (2012)

7. Khan, S.K., Yu, S., Liu, H., Deformation induced anisotropic responses of Ti-6Al-4V alloy. Part II: a strain rate and temperature dependent anisotropic yield function, International Journal of Plasticity $38,14-26(2012)$

8. Hill, R., A theory of the yielding and plastic flow of anisotropic metals", Proc. Roy. Soc. London, A193, 281-297 (1948)

9. Stoughton, T. B. and Yoon, J. W., A pressure-sensitive yield criterion under a non-associated flow rule for sheet metal forming, Int. J. Plasticity, 20, 705-731 (2004)

10. Stoughton, T. B., and Yoon, J.W., A Review of Drucker's Postulate and the Issue of Plastic Stability in Metal Forming, Int. J. of Plasticity, 22, 391-433 (2006)

11. Stoughton, T. B. and Yoon, J. W., On the existence of indeterminate solutions to the equations of motion under non-associated flow, Int. J. Plasticity, 24, 583-613 (2008)

12. Spitzig, W.A., Richmond, O., The effect of pressure on the flow stress of metals, Acta Metallurgica $32,457-463$ (1984)

13. Spitzig, W.A., Sober, R.J., Richmond, O., Pressure dependence of yielding and associated volume expansion in tempered martensite, Acta Metallurgica 23, 885-893 (1975)

14. Drucker, D.C., Prager, W., Soil mechanics and plastic analysis for limit design, Quarterly of Applied Mathematics 10, 157-165 (1952)

15. Plunkett, B., Cazacu, O., Lebensohn, R.A., Barlat, F., Elastic-viscoplastic anisotropic modeling of textured metals and validation using the Taylor cylinder impact test, International Journal of Plasticity 23, 1001-1021 (2007) 
16. Lebensohn, R.A., Tomé, C.N., A self-consistent anisotropic approach for the simulation of plastic deformation and texture development of polycrystals: application to zirconium alloy, Acta Metallurgica et Materialia 41, 2611-2624 (1993)

17. Tomé, C.N., Maudlin, P.J., Lebensohn, R.A., Kaschner, G.C., Mechanical response of zirconium-I. Derivation of a polycrystall constitutive law and finite element analysis, Acta Materialia 49, 3085-3096 (1993)

18. Jeong Whan Yoon, Yanshan Lou, Jong Hu n Yoon, and Michael V Glazoff, "Asymmetric Yield function Based on the Stress Invariants for Pressure Sensitive Metals", submitted to International Journal of Plasticity (July 15, 2013) 